\title{
Autoimmune hepatitis: review of histologic features included in the simplified criteria proposed by the international autoimmune hepatitis group and proposal for new histologic criteria
}

\author{
Dana Balitzer ${ }^{1}$, Nafis Shafizadeh ${ }^{2}$, Marion G Peters ${ }^{3}$, Linda D Ferrell ${ }^{1}$, Najeeb Alshak ${ }^{2}$ and \\ Sanjay Kakar ${ }^{1}$ \\ ${ }^{1}$ Department of Anatomic Pathology, University of California Medical Center, University of California, \\ San Francisco, CA, USA; ${ }^{2}$ Kaiser Permanente, Woodland Hills Medical Center, San Francisco, CA, USA and \\ ${ }^{3}$ Department of Medicine, University of California, San Francisco, CA, USA
}

\begin{abstract}
Simplified criteria for diagnosis of autoimmune hepatitis are based on autoantibodies, serum immunoglobulin G, histologic features, and negative viral serology. A score of 6 points is necessary for the designation of probable autoimmune hepatitis and 7 points or more for definite autoimmune hepatitis. The presence of three histologic features is required for categorizing a case as typical (2 points): interface hepatitis with portal lymphocytic/ lymphoplasmacytic cells extending into lobule, emperipolesis, and rosettes. In the absence of all three features, a chronic hepatitis picture is considered compatible with autoimmune hepatitis (1 point). This study examines the validity of these histologic features for the diagnosis of autoimmune hepatitis. Clinical data and liver biopsies were reviewed for 88 autoimmune hepatitis, 20 primary biliary cholangitis, and 13 non-autoimmune acute hepatitis cases. Interface/lobular activity, number of plasma cells, copper/CK7 stains, and presence/absence of biliary features were assessed in autoimmune hepatitis and primary biliary cholangitis cases. The simplified criteria score was calculated. Modified histologic criteria were formulated on the basis of interface/lobular activity, number of plasma cells, and presence/absence of biliary features. Using the proposed histologic features, histologic score of 2 increased from 8 to $77 \%$, while total simplified score of $>6$ increased from 69 to $86 \%$. There was no increase in total simplified score for primary biliary cholangitis or non-autoimmune acute hepatitis. Rosettes and emperipolesis are difficult to interpret, and lack sensitivity and sensitivity for autoimmune hepatitis diagnosis. The current histologic criteria used in the current simplified score lead to underscoring of autoimmune hepatitis cases. The modified histologic criteria based on the inflammatory activity, extent of plasma cells, and results of copper/CK7 staining increased the histologic score in autoimmune hepatitis and led to a probable/definite diagnosis of autoimmune hepatitis in $17 \%$ of cases that would have otherwise been classified as non- autoimmune hepatitis by simplified score.
\end{abstract}

Modern Pathology (2017) 30, 773-783; doi:10.1038/modpathol.2016.267; published online 20 January 2017

The diagnosis of autoimmune hepatitis is based on a combination of clinical presentation, liver biochemical tests, serologic findings such as serum immunoglobulin $\mathrm{G}$ and autoantibodies, results of viral hepatitis work-up and histologic findings on liver biopsy. The International Autoimmune Hepatitis

Correspondence: Dr S Kakar, MD, Department of Anatomic Pathology, University of California Medical Center, University of California, 505 Parnassus Avenue, San Francisco, CA 94143, USA. E-mail: sanjay.kakar@ucsf.edu

Received 18 October 2016; revised 17 December 2016; accepted 19 December 2016; published online 20 January 2017
Group originally proposed criteria for diagnosis of autoimmune hepatitis in $1992,{ }^{1}$ which were subsequently revised in $1999 .{ }^{2}$ The Revised criteria comprise 12 parameters, and are too complex for routine use. ${ }^{3-5}$ In addition to being cumbersome, these criteria require serologic tests that are expensive and not widely available. ${ }^{3}$ In 2008 , the International Autoimmune Hepatitis Group proposed simplified criteria that incorporate four parameters: ${ }^{3}$ autoantibodies, serum immunoglobulin G, liver biopsy findings, and results of viral hepatitis work-up (Table 1). A score of 6 is considered as 
Table 1 Simplified criteria proposed by International Autoimmune Hepatitis Group for diagnosis of autoimmune hepatitis

\begin{tabular}{|c|c|c|}
\hline Variable & Cutoff & Points \\
\hline Antinuclear antibody or smooth muscle antibody & Equal to or greater than 1:40 & 1 \\
\hline Antinuclear antibody or smooth muscle antibody & Equal to or greater than $1: 80$ & $2^{\mathrm{a}}$ \\
\hline Or liver-kidney-microsomal antibody & Equal to or greater than $1: 40$ & \\
\hline Or soluble liver antigen & Positive & \\
\hline \multirow[t]{2}{*}{ Serum immunoglobulin G } & More than upper normal limit & 1 \\
\hline & More than 1.10 times the upper normal limit & 2 \\
\hline \multirow[t]{2}{*}{ Liver histology (evidence of hepatitis is a necessary condition) } & Compatible with autoimmune hepatitis & 1 \\
\hline & Typical for autoimmune hepatitis & 2 \\
\hline Absence of viral hepatitis & Yes & 2 \\
\hline
\end{tabular}

Equal to or more than 6 points: probable autoimmune hepatitis. Equal to or more than 7 points: definite autoimmune hepatitis.

${ }^{a}$ Addition of points achieved for all autoantibodies (maximum, 2 points).

'probable autoimmune hepatitis', and a score of 7 as 'definite autoimmune hepatitis'.

Several studies have shown that the simplified criteria retain high specificity for diagnosis of autoimmune hepatitis exceeding 95\% for both scores $>6$ and $>7.3 .5$ The sensitivity for diagnosis of autoimmune hepatitis is somewhat limited, with reported numbers ranging from 65 to $95 \%$ for probable autoimmune hepatitis (score $>6$ ) and from 15 to $87 \%$ for definite autoimmune hepatitis (score $>7$ ). ${ }^{3,4,6}$ The International Autoimmune Hepatitis Group criteria do not perform well in making the diagnosis of autoimmune hepatitis in specific situations such as cases with acute presentation, ${ }^{5,7,8}$ overlap syndromes, ${ }^{5,9,10}$ and in the setting of concurrent diseases like nonalcoholic fatty liver disease. ${ }^{11}$ Autoimmune hepatitis with acute presentation clinically present as acute hepatitis; a subset of these cases show histologic features of chronicity such as fibrosis on biopsy, while others resemble acute hepatitis on histology as well. ${ }^{5,7,8,12}$

Histologic features have a critical role in the diagnosis of autoimmune hepatitis, and identification of a hepatitic pattern of injury is considered a pre-requisite for applying the simplified criteria. ${ }^{3}$ As per the simplified criteria, the biopsy findings are considered typical ( 2 points), compatible (1 point), and atypical ( 0 points). Interface hepatitis with lymphocytic/lymphoplasmacytic infiltrate, rosettes, and emperipolesis are regarded as typical of autoimmune hepatitis, and all three features have to be present to obtain a histologic score of 2 points. ${ }^{3}$ If all features considered typical are not present, a picture of chronic hepatitis with lymphocytic infiltration is regarded as being compatible (1 point) with the diagnosis. When histologic features of another disease process such as biliary diseases and steatohepatitis are identified, the histology is considered atypical (0 points).

The validity of the histologic features included in the simplified score has not been examined in detail. Rosettes are a nonspecific regenerative feature that can be seen following hepatocellular injury of any etiology and can be seen in non-autoimmune hepatitis cases. ${ }^{13,14}$ Similarly, emperipolesis is a characteristic feature of autoimmune hepatitis but is not specific. ${ }^{14,15}$ In addition to problems of specificity, both these features have modest sensitivity at best for autoimmune hepatitis cases. ${ }^{7,13,14,16}$ The presence of emperipolesis is best evaluated by confocal or electron microscopy and it is difficult to reliably assess this feature in routine diagnostic practice with light microscopy. ${ }^{2,8,15}$ In our experience, both these features are rarely mentioned in pathology reports in the United States, even in large specialized academic centers. This suggests that most cases of otherwise characteristic autoimmune hepatitis would only get a histologic score of 1 point in the absence of rosettes and emperipolesis, as both these features are required along with interface activity for a histologic score of 2 points. This histologic underscoring may account for the low sensitivity of the simplified score in some cases, as well as explain the low performance of the score for cases with acute presentation.

Copper is excreted in the bile and periportal copper deposition is seen in $50-94 \%$ of chronic cholestastic diseases. ${ }^{17,18}$ Hepatocytes in normal liver are negative for CK7, while periportal hepatocytes acquire a CK7-positive biliary phenotype in at least $70 \%$ of biopsies with chronic cholestatic disease. ${ }^{19-21}$ As periportal copper and periportal CK7-positive hepatocytes are not typically seen in hepatitic diseases like autoimmune hepatitis, negative results for copper and CK7 stains favor hepatitic over biliary patterns of injury. These stains are currently not included in the histologic scoring of autoimmune hepatitis.

The goal of this study is to critically examine the histologic features included in the simplified criteria for the diagnosis of autoimmune hepatitis, and explore modified histologic criteria that can improve the performance of the score. The study also examines the utility of incorporating the results of copper and CK7 stains in the histologic criteria. 


\section{Materials and methods}

\section{Case Selection}

Cases were selected from the pathology files of University of California San Francisco and Kaiser Permanente, Los Angeles, and included autoimmune hepatitis $(n=88)$, primary biliary cholangitis $(n=20)$, and non-autoimmune acute hepatitis $(n=13)$. All patients underwent biopsies between 1990 and 2015. The patient charts were reviewed and the following information was recorded: age, gender, medication history, autoantibody titers (smooth muscle antibodies, anti- actin antibodies, antinuclear antibodies, antimitochondrial antibodies), immunoglobulin levels (immunoglobulin G, immunoglobulin $M$ ), viral hepatitis status, biochemical tests at the time of biopsy (alanine aminotransferase, aspartate aminotransferase, alkaline phosphatase, bilirubin). All selected cases including lacked serological evidence of viral hepatitis B or C, or excessive alcohol intake. There were no study cases with coexisting steatohepatitis or Wilson disease. Drug etiology was excluded in autoimmune hepatitis cases based on history, clinical setting, response to therapy, and disease course. The diagnosis of autoimmune hepatitis was based on a combination of clinical, serologic and histologic features. Response to immunosuppressive therapy and confirmation of the clinical diagnosis during follow-up was used to select autoimmune hepatitis cases, including cases with acute presentation (follow-up range 1 month to 13 years, average 4.5 years). Primary biliary cholangitis was diagnosed on the basis of a combination of antimitochondrial antibodies positivity, chronic cholestatic biochemical profile, and/or supportive histologic features as per the American Association for the Study of Liver Diseases' criteria. ${ }^{22}$ Druginduced liver injury was excluded in all autoimmune hepatitis and primary biliary cholangitis cases.

\section{Histologic Evaluation}

The presence of rosettes, emperipolesis, and plasma cells were noted in a semiquantitative fashion. Rosettes were defined as hepatocytes arranged around a clearly identifiable luminal space (Figure 1a). Emperipolesis was defined as the presence of lymphocytes or plasma cells within the cytoplasm of hepatocytes, as best judged based on light microscopic evaluation. The feature was scored as present only when lymphocytes or plasma cells appeared intact, whereas nuclear fragments within the hepatocyte cytoplasm were ignored. Rosettes and emperipolesis were recorded as absent or present (few: $<2$ foci, many $>2$ foci). The number of plasma cells was recorded as absent, few ( $<2$ in most portal/lobular areas), moderate (2-4 in most portal/lobular areas), or abundant ( $>5$ in in portal/lobular areas). A plasma cell cluster was defined as $>5$ plasma cells at one focus. The presence of lymphocytes, eosinophils and neutrophils was similarly recorded. The inflammatory grade was determined for each case using the Ishak grading scheme ${ }^{23}$ (Table 2): interface activity (A0-A4), confluent necrosis (B1-B6), lobular necroinflammatory activity (C0-C4), and portal inflammation (D0-D4). The fibrosis was recorded using the Batts-

\section{a}

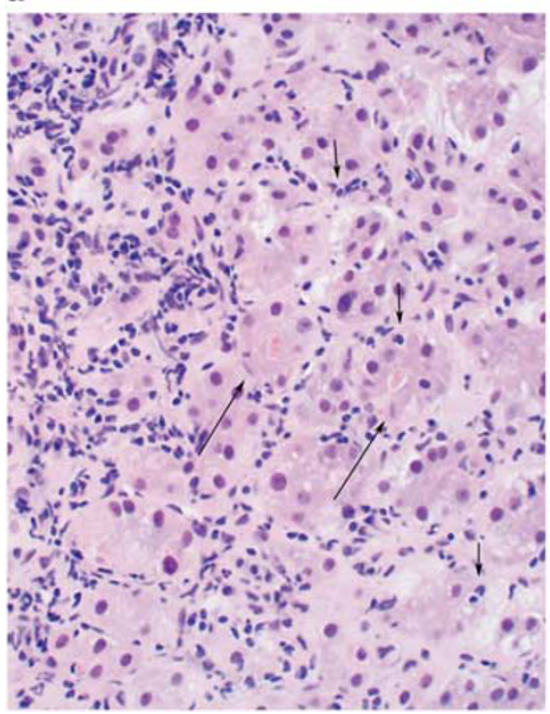

b

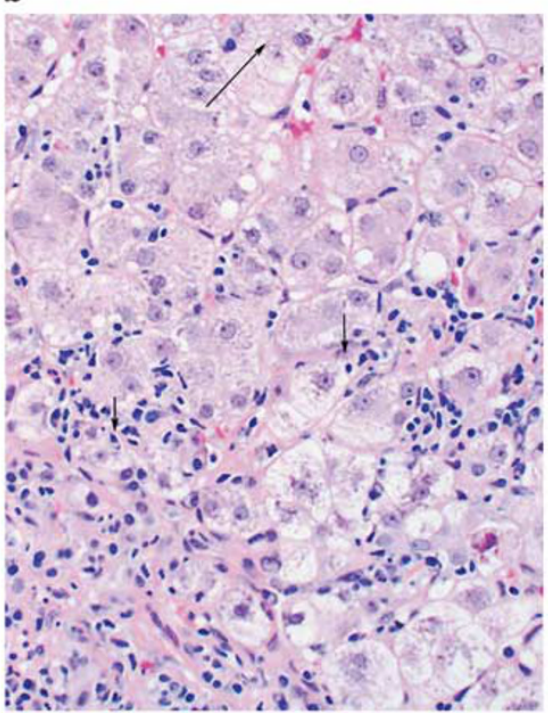

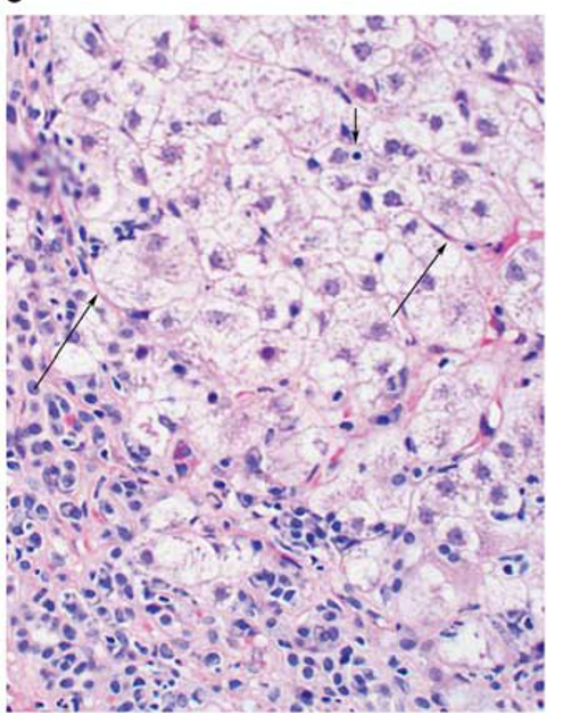

Figure 1 Histologic score in autoimmune hepatitis based on simplified criteria. Both rosettes (long arrow) and emperipolesis (short arrow) are present and would be considered as typical histology, 2 points under simplified criteria; this was observed in around one-fourth of autoimmune hepatitis cases $(\mathbf{a}, \mathrm{H} \& \mathrm{E}, \times 400)$. Autoimmune hepatitis case with emperipolesis (short arrow) and rosettes (long arrow) with small inconspicuous lumina, which can be easily overlooked (b, H\&E, $\times 400)$. Autoimmune hepatitis case with plasma cell clusters and emperipolesis is assigned a histology score of 1 point due to absence of well-defined rosettes; clusters of hepatocytes indicating regenerative activity are present (long arrow) but lack clearly defined lumina (c, H\&E, × 400). 
Ludwig scheme (scale 0-4). ${ }^{24}$ The presence of bile duct injury (absent, focal) and granulomas were recorded.

Rubeanic acid stain for copper (66 autoimmune hepatitis, 18 primary biliary cholangitis) and CK7 immunohistochemistry (63 autoimmune hepatitis, 19 primary biliary cholangitis) were done in autoimmune hepatitis cases. The results for copper stain were recorded as negative or positive based on granular cytoplasmic staining in periportal hepatocytes. The results for CK7 stain were recorded as positive if at least three periportal hepatocytes were positive in at least two foci; all other cases were scored as negative.

\section{Autoimmune Hepatitis Scoring}

The total simplified score as well as the histologic score was calculated for each case using the 2008 International Autoimmune Hepatitis Group criteria. ${ }^{3}$ The total simplified score and the histologic score were also calculated using the modified histologic criteria proposed in the study, and the results were compared with the International Autoimmune Hepatitis Group score.

\section{Statistical Analysis}

The categorical data were analyzed using the Fisher exact test.

\section{Results}

\section{Clinical Characteristics}

Of the 88 autoimmune hepatitis cases, 76 (86\%) were women. The average age at diagnosis was 42.8 years (range 11-70). The mean alanine aminotransferase and aspartate aminotransferase for autoimmune hepatitis cases was 585 U/L (range 49-2382) and $595 \mathrm{U} / \mathrm{L}$ (range 34-2267), respectively. Of the 88 autoimmune hepatitis cases, $50(57 \%)$ had an elevated alkaline phosphatase (range 63-334 U/L). Antinuclear antibodies, smooth muscle antibodies, and elevated immunoglobulin $\mathrm{G}$ were present in 69 $(78 \%), 64(73 \%)$, and $63(71 \%)$ autoimmune hepatitis cases, respectively. The median antinuclear antibody titer was 1:320 (range negative to 1:2560), and the median immunoglobulin $G$ levels were $2260 \mathrm{mg} / \mathrm{mL}$ or 1.28 times the upper limit of normal (range 1230-6680). Liver-kidney microsome antibodies were positive in 1 of $30(3 \%)$ cases tested. There were no seronegative autoimmune hepatitis cases.

All 20 primary biliary cholangitis cases occurred in women with an average age of 49 years (range 11-68). Alkaline phosphatase was elevated in 17 (85\%) cases (range 53-401 U/L). The mean alanine aminotransferase and aspartate aminotransferase for cases of primary biliary cholangitis was $81 \mathrm{U} / \mathrm{L}$ (range 26-503) and 84 U/L (range 22-310)
Table 2 Ishak grading scheme

\begin{tabular}{ll} 
Description & Score \\
\hline $\begin{array}{l}\text { A. Periportal or periseptal interface hepatitis (piecemeal } \\
\text { necrosis) }\end{array}$ & \\
None & A0 \\
Mild (focal, few portal areas) & A1 \\
Mild/moderate (focal, most portal areas) & A2 \\
Moderate (continuous around <50\% of tracts or septa) & A3 \\
Severe (continuous around $>50 \%$ of tracts or septa) & A4 \\
& \\
B. Confluent necrosis & B0 \\
None & B1 \\
Focal confluent necrosis & B2 \\
Zone 3 necrosis in some areas & B3 \\
Zone 3 necrosis in most areas & B4 \\
Zone 3 necrosis+occasional portal-central bridging & B5 \\
Zone 3 necrosis+multiple portal-central bridging & B6 \\
Panacinar or multiacinar necrosis & \\
& \\
C. Focal (spotty) lytic necrosis, apoptosis and focal & C0 \\
inflammation & C1 \\
None & C2 \\
One focus or less per $\times 10$ objective & C3 \\
Two to four foci per $\times 10$ objective & C4 \\
Five to ten foci per $\times 10$ objective & \\
More than 10 foci per $\times 10$ objective & D0 \\
D. Portal inflammation & D1 \\
None & D2 \\
Mild, some or all portal areas & D3 \\
Moderate, some or all portal areas & D4 \\
Moderate/marked, all portal areas & \\
Marked, all portal areas & \\
\hline
\end{tabular}

respectively. Serum immunoglobulin $M$ was elevated in 7 of 16 cases (44\%). Antimitochondrial antibodies were positive in 16 cases (80\%). Smooth muscle antibodies were positive in 8 of 19 cases $(42 \%)$, and antinuclear antibodies were positive in 5 of 18 cases (28\%); the results for smooth muscle antibody and antinuclear antibody were not available for one and two patients, respectively. Liverkidney microsome antibodies were not tested. The diagnosis of primary biliary cholangitis was confirmed by clinical and histologic features and clinical course of disease.

Of the 13 non-autoimmune acute hepatitis cases, 10 were women (mean age 44 years, range 2-70). These included viral hepatitis ( $n=2$, one hepatitis A, one hepatitis $\mathrm{C})$ and adverse drug reaction $(n=3$, one each due to nitrofurantoin, an antidepressant, and an over-the-counter arthritis medication 'Move Free'). The etiology was not known in the remaining eight cases. The diagnosis of drug-induced acute hepatitis was based on temporal profile of drug ingestion and onset of disease, exclusion of other diseases, and liver biopsy findings. Antimitochondrial antibodies were negative in all 11 cases that were tested, smooth muscle antibody was positive in 3 of $13(27 \%)$ cases, and antinuclear antibodies were positive in 4 of $13(31 \%)$ cases. Alkaline phosphatase was elevated in 4 of $13(31 \%)$ cases, and the mean alanine 
aminotransferase and aspartate aminotransferase was 904 U/L (range 134-1960) and $817 \mathrm{U} / \mathrm{L}$ (range 189-2562), respectively. Elevated immunoglobulin $\mathrm{G}$ (range $867-3200 \mathrm{mg} / \mathrm{mL}$ ) was observed in two $(15 \%)$ cases, both of which lacked autoantibodies and were self-limited. Autoimmune hepatitis was excluded in these cases based on the clinical and lab findings as well as lack of autoimmune hepatitis-like disease course on follow-up.

\section{Pathologic Characteristics}

Emperipolesis was identified in 57 (65\%) autoimmune hepatitis cases, and was also present in 10 $(50 \%)$ primary biliary cholangitis cases $(P=0.31)$ and $10(77 \%)$ non-autoimmune acute hepatitis cases $(P=0.53$; Figures 1 and 2; Table 3$)$. Rosettes were present in $29(33 \%)$ autoimmune hepatitis cases, which was similar to 5 (38\%) non-autoimmune acute hepatitis cases $(P=0.78$; Figure 2$)$. Rosettes were not identified in any primary biliary cholangitis case. Both emperipolesis and rosettes were identified in $23(26 \%)$ of autoimmune hepatitis cases and $4(31 \%)$ of non-autoimmune acute hepatitis cases $(P=1.0)$.

Plasma cell clusters (defined as $>5$ cells) were identified in $49(56 \%)$ autoimmune hepatitis cases compared with seven (35\%) primary biliary cholangitis cases $(P=0.14)$ and two $(15 \%)$ nonautoimmune acute hepatitis $(P=0.008$; Table 3$)$. Abundant plasma cells were identified significantly more often in autoimmune hepatitis (66/88, 75\%) compared with primary biliary cholangitis $(10 / 20$, $50 \%, P=0.03)$ and non-autoimmune acute hepatitis $(4 / 13,31 \%, P=0.04)$.

Interface activity (Ishak A score $>2$ ) was present in $70(80 \%)$ autoimmune hepatitis cases compared with $10(77 \%)$ non-autoimmune acute hepatitis $(P=0.73)$ and three $(15 \%)$ primary biliary cholangitis cases $(P<0.001)$. Confluent necrosis (Ishak B scores $>2$ ) was present in $35(40 \%)$ autoimmune hepatitis cases compared with nine (69\%) non-autoimmune acute hepatitis $(P=0.07)$ and none of the primary biliary cholangitis cases $(P<0.001)$. Lobular necroinflammatory activity (Ishak C score $>2$ ) was observed in $66(75 \%)$ autoimmune hepatitis cases compared with 12 (92\%) non-autoimmune acute hepatitis $(P=0.29)$ and three $(15 \%)$ primary biliary cholangitis cases $(P<0.001)$. Ishak $D$ scores $>2$ were present in $79(90 \%)$ autoimmune hepatitis cases compared with 11 (85\%) non-autoimmune acute hepatitis $(P=0.63)$ and six $(30 \%)$ primary biliary cholangitis cases $(P<0.001)$. In six autoimmune hepatitis cases $(7 \%)$, there was lobular activity (C2 or higher) without significant interface activity a

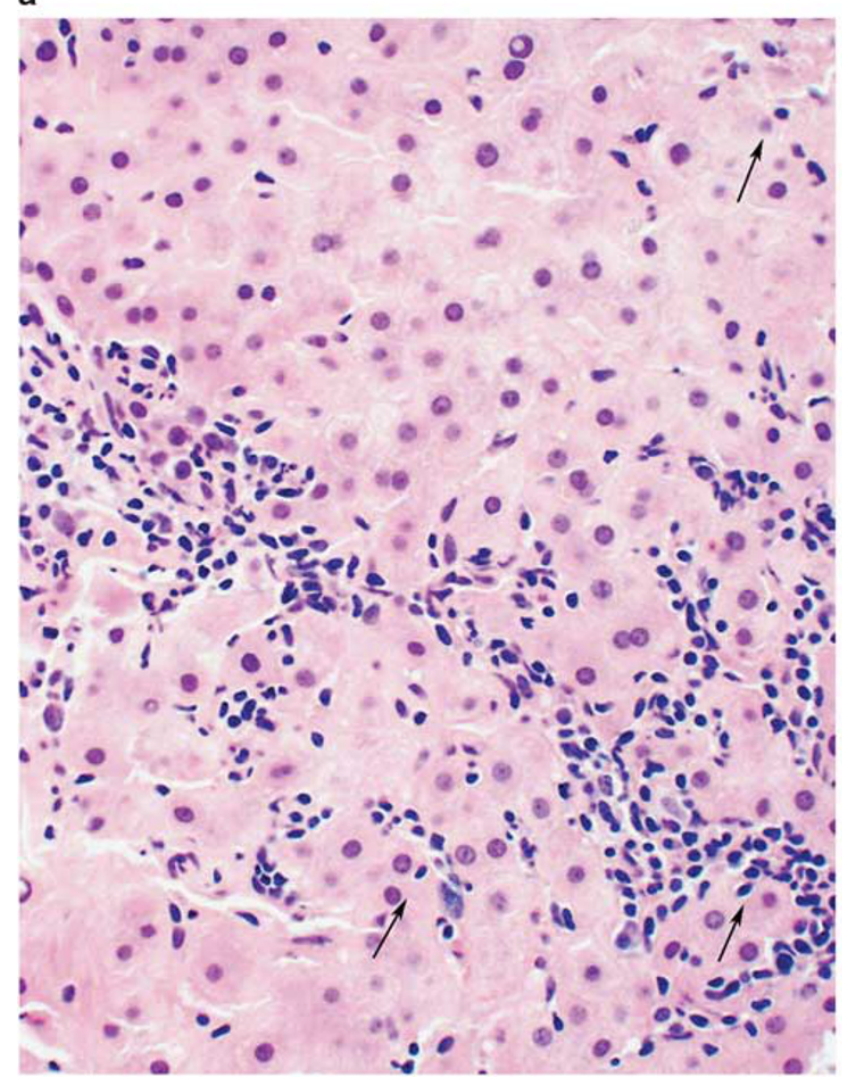

b

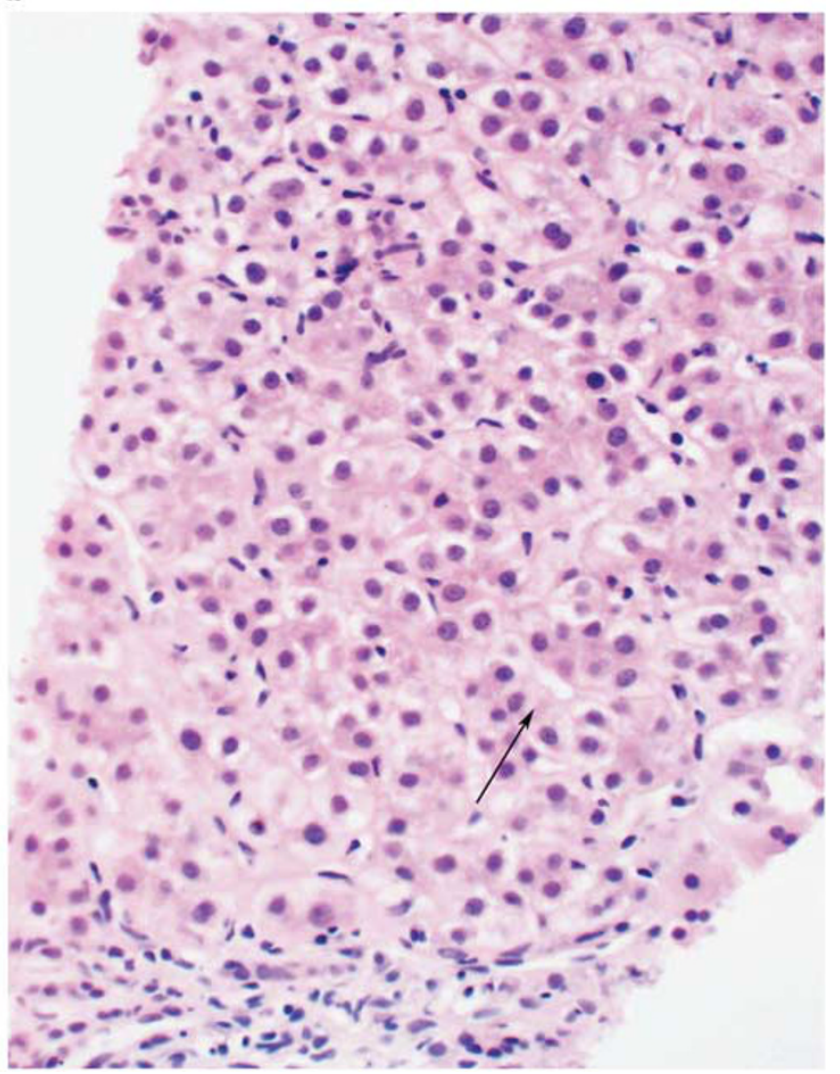

Figure 2 Emperipolesis in primary biliary cholangitis $(\mathbf{a}, \mathrm{H} \& \mathrm{E}, \times 400)$, and rosettes in non-autoimmune acute hepatitis $(\mathbf{b}, \mathrm{H} \& \mathrm{E}, \times 200)$. 
Table 3 Pathologic characteristics of autoimmune hepatitis, primary biliary cholangitis and non-autoimmune acute hepatitis

\begin{tabular}{|c|c|c|c|c|}
\hline & $\begin{array}{l}\text { Autoimmune } \\
\text { hepatitis } \\
(\mathrm{n}=88)\end{array}$ & $\begin{array}{l}\text { Primary biliary } \\
\quad \text { cholangitis } \\
\quad(\mathrm{n}=20)\end{array}$ & $\begin{array}{l}\text { Non-autoimmune } \\
\text { acute hepatitis } \\
\quad(\mathrm{n}=13)\end{array}$ & $\begin{array}{l}\text { P-value (autoimmune hepatitis vs } \\
\text { primary biliary cholangitis, autoimmune } \\
\text { hepatitis vs non-autoimmune acute) }\end{array}$ \\
\hline Emperipolesis & $57(65)$ & $10(50)$ & $10(77)$ & $\begin{array}{l}0.31 \\
0.53\end{array}$ \\
\hline Rosettes & $29(33)$ & 0 & $5(38)$ & 0.78 \\
\hline Plasma cells (any number) & 87 (99) & $14(70)$ & $11(85)$ & $\begin{array}{r}<0.001 \\
0.044\end{array}$ \\
\hline Numerous plasma cells & $43(49)$ & $4(20)$ & $1(8)$ & $\begin{array}{l}0.024 \\
0.006\end{array}$ \\
\hline Plasma cell clusters & $49(56)$ & $7(35)$ & $2(15)$ & $\begin{array}{l}0.582 \\
0.008\end{array}$ \\
\hline Interface activity & & & & \\
\hline $\begin{array}{l}\text { Ishak score }<\text { A1 } \\
\text { Ishak score }>\text { A2 }\end{array}$ & $\begin{array}{l}18(20) \\
70(80)\end{array}$ & $\begin{aligned} & 20(100) \\
& 3(15)\end{aligned}$ & $\begin{array}{c}3(23) \\
10(77)\end{array}$ & $\begin{array}{r}<0.001 \\
0.730\end{array}$ \\
\hline $\begin{array}{l}\text { Confluent necrosis } \\
\text { Ishak score }<\text { B1 } \\
\text { Ishak score }>\text { B2 }\end{array}$ & $\begin{array}{l}55(63) \\
33(37)\end{array}$ & $\begin{array}{l}20(100) \\
0(0)\end{array}$ & $\begin{array}{l}4(40) \\
9(60)\end{array}$ & $\begin{array}{r}<0.001 \\
0.014\end{array}$ \\
\hline $\begin{array}{l}\text { Lobular activity } \\
\text { Ishak score < C1 } \\
\text { Ishak score }>\mathrm{C} 2\end{array}$ & $\begin{array}{l}24(27) \\
64(73)\end{array}$ & $\begin{array}{l}20(100) \\
3(15)\end{array}$ & $\begin{array}{c}1(8) \\
12(92)\end{array}$ & $\begin{array}{r}<0.001 \\
0.177\end{array}$ \\
\hline $\begin{array}{l}\text { Portal inflammation } \\
\text { Ishak score }<\text { D2 } \\
\text { Ishak score }>\text { D3 }\end{array}$ & $\begin{array}{l}46(52) \\
42(48)\end{array}$ & $\begin{array}{c}20(100) \\
0\end{array}$ & $\begin{array}{l}8(62) \\
5(38)\end{array}$ & $\begin{array}{r}<0.001 \\
0.568\end{array}$ \\
\hline Positive periportal copper stain & $2 / 39(5)$ & $11 / 21(52)$ & $0 / 2$ & $\begin{array}{r}<0.001 \\
1.000\end{array}$ \\
\hline Positive periportal CK7 stain & 7/38 (18) & $13 / 21(62)$ & $0 / 1$ & $\begin{array}{l}0.001 \\
1.000\end{array}$ \\
\hline
\end{tabular}

Figures in parenthesis reflect percentages.

(A1 or lower). Fibrosis was present in $57(67 \%)$ autoimmune hepatitis and eight $(27 \%)$ primary biliary cholangitis cases (Table 3).

\section{Copper and CK7 Stains}

Copper stain was positive in 2/39 (5\%) autoimmune hepatitis cases and 9/18 (50\%) primary biliary cholangitis cases $(P<0.001$; Figure 3$)$. Both copperpositive autoimmune hepatitis cases had advanced fibrosis with bridging (stage 3). CK7 staining in periportal hepatocytes was observed in $7 / 38(18 \%)$ autoimmune hepatitis cases and 12/19 (63\%) primary biliary cholangitis cases $(P=0.001)$. Of the seven cases of autoimmune hepatitis with positive CK7 staining, three showed periportal fibrous septa with rare thin bridges and were categorized as stage 2 as per Batts-Ludwig scheme, while the remaining showed established bridging fibrosis (stage 3).

\section{Simplified International Autoimmune Hepatitis Group Score}

Simplified score of $>6$ was observed in 66 (75\%) autoimmune hepatitis cases. A score of $>6$ was also observed in three (23\%) non-autoimmune acute hepatitis as well as three primary biliary cholangitis cases $(15 \%)$. For cases of autoimmune hepatitis, seven $(8 \%)$ had a histologic score of $0,58(66 \%)$ had a score of 1 , and $23(26 \%)$ had a score of 2 . For primary biliary cholangitis cases, $18(90 \%)$ had a score of 0 , two $(10 \%)$ had a score of 1 , and none had a score of 2. For non-autoimmune hepatitis acute hepatitis cases, none had a score of 0 , eight $(62 \%)$ had a score of 1 , and five (38\%) had a score of 2 .

\section{Modified Histologic Criteria and Reassessment of Autoimmune Hepatitis Score}

Modified histologic criteria were formulated (Table 4) based on the inflammatory activity, extent of plasma cells, and results of copper/CK7 staining. A histologic score of 1 was assigned for cases with mild or moderate necroinflammatory activity (Ishak scores of at least A2, B1, and/or C2) and negative staining for copper/CK7 (latter applicable only for cases with Batts-Ludwig fibrosis stage $<2$ ). Copper and CK7 are not required for acute cases as chronic biliary disease is not in the differential diagnosis. A histologic score of 2 is assigned for cases with marked necroinflammatory activity (Ishak activity 
a

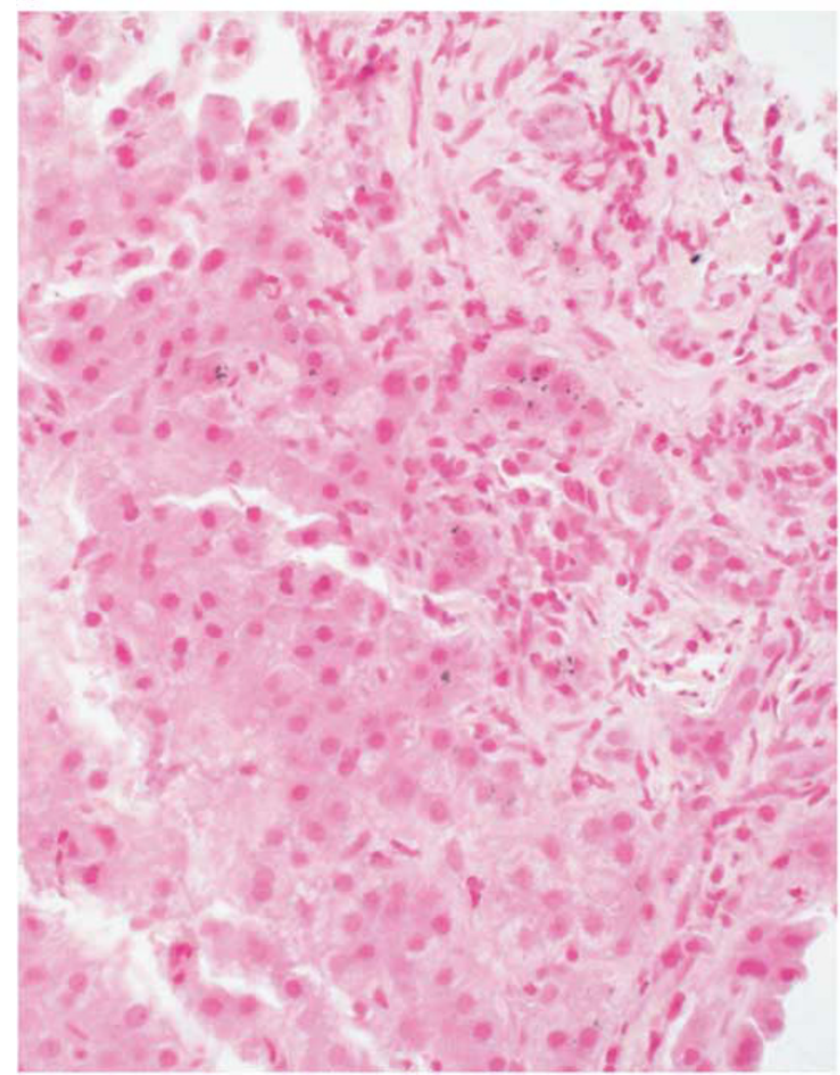

b

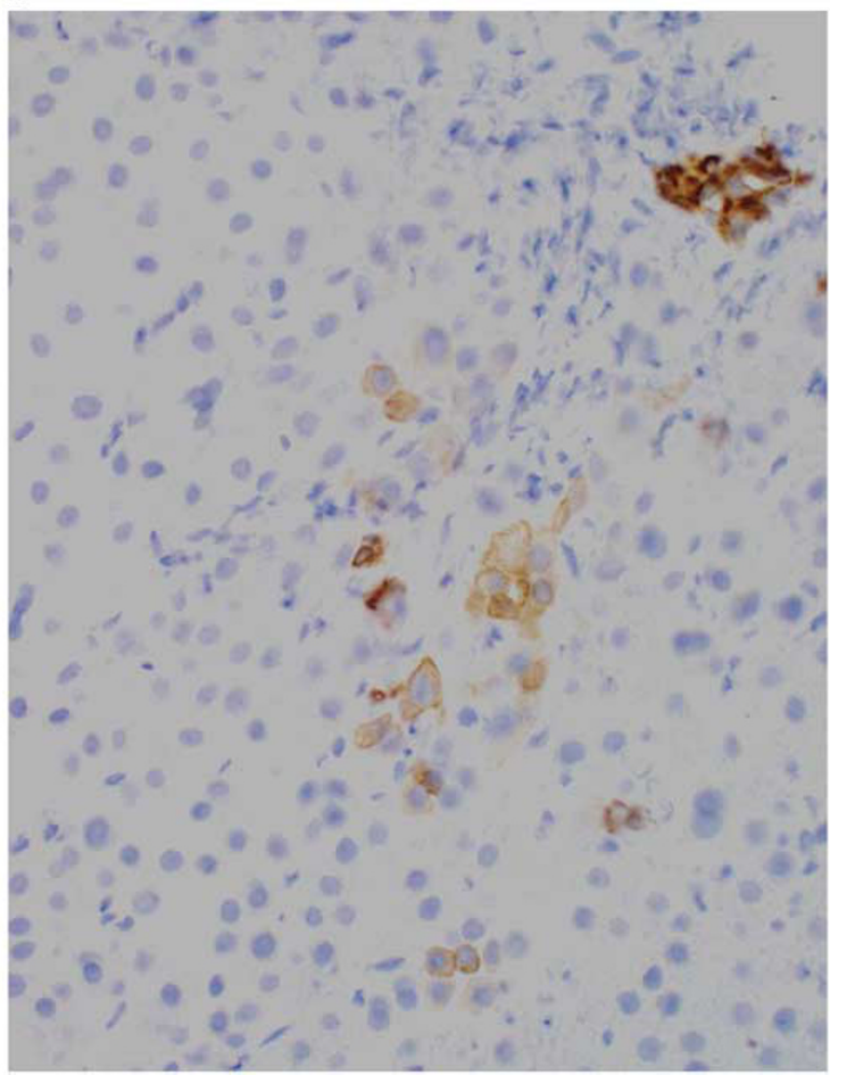

Figure 3 Periportal copper in hepatocytes (a, rubeanic acid stain, $\times 400$ ), and staining of periportal hepatocytes with CK7 (b, CK7 immunohistochemistry, $\times 200$ ) in primary biliary cholangitis.

Table 4 Proposed criteria for the histologic scoring of autoimmune hepatitis

Histology score 0

Features not observed in autoimmune hepatitis: florid duct lesion (primary biliary cholangitis), bile duct loss, or copper/CK7 positivity (latter applicable only in cases without any bridging fibrosis)

Histologic score $1^{\mathrm{a}}$

(1) Hepatitis with mild or moderate necroinflammatory activity with any of the following:

(a) Ishak A2 (mild/moderate interface activity)

(b) Ishak B1 (focal confluent necrosis)

(c) Ishak C2 (2-4 foci of lobular activity per $\times 10)$

(2) CK7 and copper stains negative (applicable only for cases with Ishak fibrosis score $<3$, this feature is not applicable to acute cases)

\section{Histologic score 2}

Hepatitic picture with any of the following:

(1) Plasma cells: numerous or in clusters

(2) High necroinflammatory activity featuring at least one of the following:

(a) Ishak score A3 or higher (at least moderate interface activity)

(b) Ishak B2 or higher (confluent necrosis in zone 3 or beyond)

(c) Ishak C3 or higher (5 or more foci of lobular activity per $\times 10$ )

${ }^{\mathrm{a} B o t h}(1)$ and (2) are necessary for histologic score of 1 except in cases with acute presentation when biliary disease is not a consideration and these stains are not relevant.

scores of at least $\mathrm{A} 3$, B2, and/or C3), numerous plasma cells or plasma cell clusters (Figure 4). Cases with features of biliary disease like prominent lymphocytic bile duct injury, florid duct lesion, bile duct loss, and copper/CK7 staining (latter applicable to Batts-Ludwig stage $<2$ only) were assigned a histologic score of 0 .
Using the modified criteria, there was an increase of histologic score for autoimmune hepatitis cases: $68(77 \%)$ with histologic score of 2 compared with $7(8 \%)$ using the simplified criteria. The total simplified score for autoimmune hepatitis increased from $<5$ (not autoimmune hepatitis) to $>6$ (probable or definite autoimmune hepatitis) in $17 \%$ of 


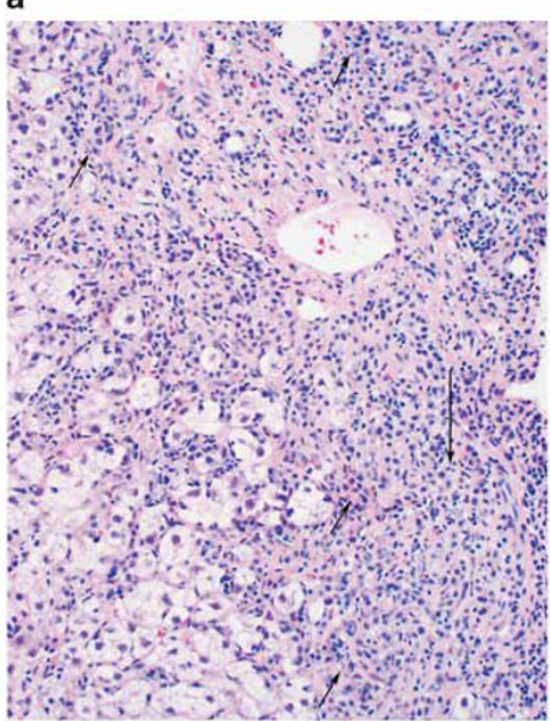

b

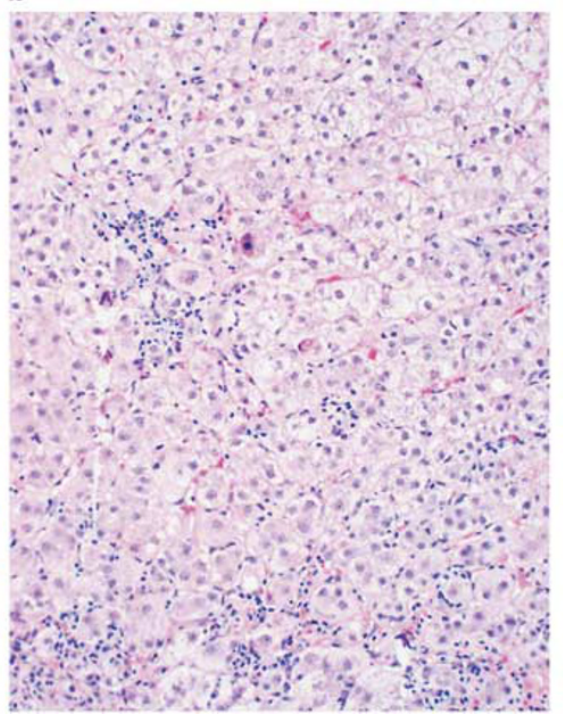

c

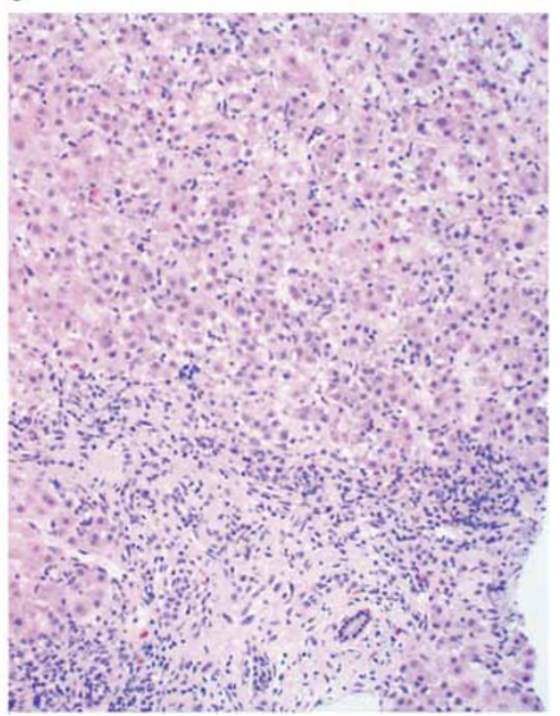

Figure 4 Histologic score based on modified histologic criteria. Autoimmune hepatitis with confluent necrosis (Ishak B4; long arrow) and prominent plasma cells (short arrow) would be assigned 2 points even in the absence of rosettes and emperipolesis (a, H\&E, $\times 200$ ). Autoimmune hepatitis with numerous foci of inflammatory lobular activity (Ishak C4) would be assigned 2 points irrespective of whether interface activity, rosettes, and emperipolesis are present or absent (b, H\&E, $\times 200)$. Autoimmune hepatitis with moderate interface (Ishak A3) and foci of inflammatory lobular activity (Ishak C3) would be assigned 2 points (c, H\&E, $\times 200$ ).

Table 5 Impact of proposed histologic score on the diagnosis of autoimmune hepatitis, primary biliary cholangitis, and non-autoimmune acute hepatitis

\begin{tabular}{|c|c|c|c|c|c|c|c|c|}
\hline & \multicolumn{4}{|c|}{ Histologic score } & \multicolumn{4}{|c|}{ Total score } \\
\hline & \multicolumn{2}{|c|}{$\begin{array}{l}\text { Simplified } \\
\text { criteria }\end{array}$} & \multicolumn{2}{|c|}{$\begin{array}{c}\text { Proposed } \\
\text { criteria }\end{array}$} & \multicolumn{2}{|c|}{$\begin{array}{l}\text { Simplified } \\
\text { criteria }\end{array}$} & \multicolumn{2}{|c|}{$\begin{array}{l}\text { Proposed } \\
\text { criteria }\end{array}$} \\
\hline & 1 & 2 & 1 & 2 & $<5$ & $>6$ & $<5$ & $>6$ \\
\hline Autoimmune hepatitis $(n=88)$ & $51 \%$ & $8 \%$ & $14 \%$ & $77 \%$ & $31 \%$ & $69 \%$ & $14 \%$ & $86 \%$ \\
\hline Primary biliary cholangitis $(n=20)$ & $9 \%$ & 0 & 0 & 0 & $85 \%$ & $15 \%$ & $85 \%$ & $15 \%$ \\
\hline Non-autoimmune acute hepatitis $(n=13)$ & $62 \%$ & $38 \%$ & $15 \%$ & $85 \%$ & $77 \%$ & $23 \%$ & $77 \%$ & $23 \%$ \\
\hline
\end{tabular}

cases (69 to $86 \%$ ) (Table 5). The histologic score increased for non-autoimmune acute hepatitis cases: $12(92 \%)$ with histologic score of 2 compared with 5 $(38.5 \%)$ using the original criteria. However, there was no increase in the total simplified score in these cases. There was no change in the histologic or total simplified score for primary biliary cholangitis cases. Three cases each of primary biliary cholangitis had interface activity (Ishak A2) and lobular activity (Ishak C2), but were assigned a histologic score of 0 due to presence of florid duct lesion (two cases), bile duct loss (two cases), and/or positive copper/CK7 (five cases).

For autoimmune hepatitis cases showing acute histology $(n=29)$, the histologic score of 2 was achieved in 27 (93\%) patients with modified criteria compared with none with simplified criteria. With the use of modified criteria, the cases with simplified score of $>6$ increased from 62 to $90 \%$ in this group.

\section{Discussion}

Liver biopsy is considered a critical element in the diagnosis of autoimmune hepatitis, and is considered an independent factor to distinguish autoimmune hepatitis from other liver diseases. ${ }^{3,6}$ Multiple studies have established the key role of liver biopsy in autoimmune hepatitis, ${ }^{3,10,16}$ and the practice guidelines of American Association for the Study of Liver Diseases as well as European Association for Study of Liver advocate the use of liver biopsy for diagnosis and treatment decisions. ${ }^{25,26}$

The simplified criteria proposed by International Autoimmune Hepatitis Group mandate the presence of three histologic features in the same biopsy for the 
case to qualify as a histologically 'typical' (2 points): interface lymphocytic/lymphoplasmacytic inflammatory activity, rosettes, and emperipolesis. Our results question the utility of including rosettes and emperipolesis as necessary features for a 'typical' designation. Both these features suffer from the problems of inconsistent histologic interpretation and modest sensitivity. The Simplified criteria as well as most studies that evaluate rosettes do not provide any criteria for morphologic evaluation..$^{7,13,16}$ Clear histologic criteria were outlined in two studies, ${ }^{14,27}$ and we have used the same features to determine the presence of rosettes, ie, arrangement of hepatocytes around a lumen. Rosettes were identified in only one-third of autoimmune hepatitis cases in our series and a similar number of nonautoimmune acute hepatitis cases. Numbers ranging from 29 to $75 \%$ have been reported in autoimmune hepatitis..,13,14,16 Rosettes have also been reported in $11 \%$ of primary biliary cholangitis, ${ }^{15} 2-41 \%$ of drug-induced liver injury, ${ }^{13,16} 23 \%$ of chronic viral hepatitis, ${ }^{14}$ and $4 \%$ of Wilson's disease cases.

In the simplified criteria, emperipolesis has been defined as 'active penetration of one cell into and through a larger cell'. The penetration of lymphocytes and/or plasma cells into hepatocytes has an important role inflicting hepatocellular injury as part of the pathogenesis of autoimmune hepatitis, but its determination by light microscopy is challenging. ${ }^{8}$ Most studies that evaluate emperipolesis do not provide any definite criteria for evaluation of this feature,${ }^{13}$ but clear criteria were used in one study. ${ }^{14}$ We used similar criteria, scoring this feature as present only when intact lymphocytes or plasma cells appeared to be in the cytoplasm of hepatocytes, while cytoplasmic nuclear fragments were not considered. Emperipolesis was seen in two-thirds of autoimmune hepatitis cases, which is slightly lower but comparable to the $75-80 \%$ range reported in literature. ${ }^{13,14,27}$ Similar numbers were observed in non-autoimmune acute hepatitis and primary biliary cholangitis cases. Hence rosettes and emperipolesis appear to have limitations in terms of both sensitivity and specificity for diagnosis of autoimmune hepatitis.

Some studies have argued that rosettes and emperipolesis are highly discriminant features and have advocated their use for the diagnosis of autoimmune hepatitis. However, a close look at the data from these studies also demonstrates the poor sensitivity of combination of rosettes and emperipolesis for autoimmune hepatitis, with a typical designation (worth 2 points on histologic component of the simplified score) in only $27 \%$ of adult cases ${ }^{14}$ and $56 \%$ (ref. 27) in pediatric cases. Despite arguments in these studies for the high specificity of rosettes and emperipolesis, their data confirm our conclusion that the continued use of these features for determining a 'typical' designation can lead to underscoring of histologic score and can contribute to the relatively low sensitivity of the simplified score. The high specificity for rosettes and emperipolesis reported by these two studies may also be related to the control groups, chronic hepatitis B in one study ${ }^{14}$ and chronic viral hepatitis/Wilson disease in the other. ${ }^{27}$ None of these studies had non-autoimmune acute hepatitis in the control group, which are more likely to show emperipolesis and rosettes. A close look at the images in the study by de Boer et $a l^{14}$ shows that rosettes with clearly defined central lumina are difficult to find even though it is stated in the figure legend that $25-50 \%$ of parenchyma is involved. It appears that clustering of hepatocytes without a clearly delineated central lumen has been interpreted as rosettes in this study, further emphasizing the inter-observer variability of this finding. Emperipolesis and rosettes have also been argued to favor autoimmune hepatitis over drug-induced liver injury. ${ }^{13}$ Although this may be true when case series are examined, their utility in an individual case is limited as these features were identified in nearly $40 \%$ of drug-induced liver injury with hepatitic pattern in the same study. ${ }^{13}$ This study had a low inter-observer agreement regarding the drug vs autoimmune hepatitis diagnosis among four pathologists $(46 \%)$. Although the reasons for discordance are not stated in the study, ${ }^{13}$ it may be related to variation in the interpretation of histologic features such as rosettes and emperipolesis. None of these studies have focused on the negative impact of missing a diagnosis of autoimmune hepatitis by requiring the presence of both rosettes and emperipolesis, features with relatively low sensitivity for autoimmune hepatitis in these studies as well as in our series.

Interface hepatitis, the third criterion used for 'typical designation in the current simplified criteria,' was present in 70 (80\%) autoimmune hepatitis cases. Lobular or centrizonal inflammatory activity with absent or minimal interface hepatitis can occur in autoimmune hepatitis ( $7 \%$ in our series) and would be missed by the current histologic features used in the simplified criteria. ${ }^{28,29}$ In our series of well characterized autoimmune hepatitis cases, only $8 \%$ had 'typical' histology as per simplified criteria and hence were awarded 2 points. This suggests that the current criteria that include emperipolesis and rosettes are contributing to underscoring of the histologic component of the criteria. This led us to formulate modified histologic criteria for inclusion in the scoring system. In the modified scheme, histologic score of 1 or 2 points was awarded based on degree of hepatitic activity (interface and/or lobular) and prominence of plasma cells. In essence, mild hepatitis with no evidence of biliary features gets 1 point, while moderate to severe hepatitis or numerous plasma cells get 2 points. As chronic biliary disease can show a pattern resembling chronic hepatitis, a histologic score of 1 required absence of cholestatic features on copper and CK7 stains. Copper accumulation and CK7-positive staining in periportal hepatocytes are recognized 
features of chronic cholestasis, ${ }^{17-21}$ and can help in the distinction of chronic hepatitic vs biliary disease. Since nonspecific copper accumulation and CK7 positivity can be seen irrespective of etiology when advanced fibrosis is present, this feature cannot be used for cases with bridging fibrosis or cirrhosis. As biliary disease is not a consideration for cases with a presentation like acute hepatitis, copper and CK7 stains are not relevant in this setting. The use of copper and CK7 stains helped in correct scoring of six primary biliary cholangitis cases that could otherwise have received a histologic score of 1 based on interface or lobular inflammatory activity.

The use of modified histologic criteria led to increase in the number of autoimmune hepatitis cases having a histologic score of 2 from 8 to $77 \%$. Using the original histologic criteria, simplified score of $>6$ was observed in $69 \%$ of autoimmune hepatitis cases, which is lower than observed in the original study but within the $65-95 \%$ range reported in literature. ${ }^{3,4,6}$ When the total simplified score was calculated on the basis of the modified histologic criteria, there was an increase of autoimmune hepatitis cases with score $>6$ to $86 \%$. In other words, $17 \%$ of autoimmune hepatitis cases that would have been labeled as non-autoimmune hepatitis based on the simplified criteria using the current histologic features would have been classified as probable or definite autoimmune hepatitis by the use of modified histologic features.

The use of the modified histologic criteria also facilitated the diagnosis of autoimmune hepatitis with histologic features of acute hepatitis, which is a major weakness in the use of simplified criteria for autoimmune hepatitis diagnosis. A histologic score of 2 was not observed in any acute autoimmune hepatitis case in our study, which is similar to the $3.6 \%$ figure reported earlier. ${ }^{8}$ In these cases, the reported sensitivity of simplified score for probable or definite autoimmune hepatitis is $0-28 \% .^{5,7,8}$ When the modified histologic criteria were used, histologic score of 2 was observed in 27 (93\%) of our cases, and the sensitivity for probable/definite autoimmune hepatitis went up from 62 to $90 \%$. The improved diagnosis of acute autoimmune hepatitis would facilitate timely immunosuppressive therapy as the high necroinflammatory activity in these cases can potentially lead to rapid progression of fibrosis if left untreated.

In our series, simplified score $>6$ was observed in three (15\%) primary biliary cholangitis cases, which is comparable to the $9 \%$ figure that has been reported. ${ }^{16}$ Neither the histology score nor the total simplified score was affected for the primary biliary cholangitis cases using the modified histologic criteria. The modified histologic criteria led to increase in the histology score of non-autoimmune acute hepatitis cases, but did not affect the total simplified score.

In summary, the currently described histologic features of rosettes and emperipolesis are difficult to interpret, and lack sensitivity and sensitivity for the diagnosis of autoimmune hepatitis. We propose modified histologic criteria using interface/lobular inflammatory activity, number of plasma cells, biliary features, and copper/CK7 stains. The use of modified histologic criteria increases the sensitivity for autoimmune hepatitis diagnosis, and can be especially helpful in acute hepatitis cases. The use of the modified histologic features can increase the sensitivity of simplified criteria for autoimmune hepatitis diagnosis, potentially leading to early diagnosis and timely institution of immunosuppressive therapy. Validation of these results in prospective series as well as in challenging situations like overlap syndrome will further help in evaluating the efficacy of modified histologic criteria.

\section{Disclosure/conflict of interest}

The authors declare no conflict of interest.

\section{References}

1 Johnson PJ, McFarlane IG. Meeting report: International Autoimmune Hepatitis Group. Hepatology 1993;18: 998-1005.

2 Alvarez F, Berg PA, Bianchi FB, et al. International Autoimmune Hepatitis Group Report: review of criteria for diagnosis of autoimmune hepatitis. J Hepatol 1999;31:929-938.

3 Hennes EM, Zeniya M, Czaja AJ, et al. Simplified criteria for the diagnosis of autoimmune hepatitis. Hepatology 2008;48:169-176.

4 Wiegard C, Schramm C, Lohse A. Scoring systems for the diagnosis of autoimmune hepatitis: past, present, and future. Semin Liver Dis 2009; 29:254-261.

5 Yeoman AD, Westbrook RH, Al-Chalabi T, et al. Diagnostic value and utility of the simplified International Autoimmune Hepatitis Group (International Autoimmune Hepatitis Group) criteria in acute and chronic liver disease. Hepatology 2009; 50:538-545.

6 Gatselis, Zachou K, Koukoulis GK, Dalekos GN. Autoimmune hepatitis, one disease with many faces: etiopathogenetic, clinico- laboratory and histological characteristics. World J Gastroenterol 2015;21: 60-83.

7 Miyake Y, Iwasaki Y, Kobashi H, et al. Clinical features of autoimmune hepatitis diagnosed based on simplified criteria of the International Autoimmune Hepatitis Group. Dig Liver Dis 2010;42:210-215.

8 Fujiwara K, Yasui S, Tawada A, et al. Diagnostic value and utility of the simplified International Autoimmune Hepatitis Group criteria in acute-onset autoimmune hepatitis. Liver Int 2011;31:1013-1020.

9 Boberg KM, Chapman RW, Hirschfield GM, et al. Overlap syndromes: the International Autoimmune Hepatitis Group (International Autoimmune Hepatitis Group) position statement on a controversial issue. J Hepatol 2011;54:374-385.

10 Papamichalis PA, Zachou K, Koukoulis GK, et al. The revised international autoimmune hepatitis score in chronic liver diseases including autoimmune hepatitis/ overlap syndromes and autoimmune hepatitis with 
concurrent other liver disorders. J Autoimmune Dis 2007;4:3.

11 Yatsuji S, Hashimoto E, Kaneda $\mathrm{H}$ et al. Diagnosing autoimmune hepatitis in nonalcoholic fatty liver disease: is the International Autoimmune Hepatitis Group scoring system useful? J Gastroenterol 2005;40:1130-1138.

12 Krawitt EL. Autoimmune hepatitis. N Engl J Med 2006;354:54-66.

13 Suzuki A, Brunt EM, Kleiner DE, et al. The use of liver biopsy evaluation in discrimination of idiopathic autoimmune hepatitis versus drug induced liver injury. Hepatology 2011;54:931-939.

14 de Boer YS, van Nieuwkerk CM, Witte BI, et al. Assessment of the histopathological key features in autoimmune hepatitis. Histopathology 2015;66: 351-362.

15 Miao Q, Bian Z, Tang R, et al. Emperipolesis mediated by CD8 $\mathrm{T}$ cells is a characteristic histopathologic feature of autoimmune hepatitis. Clin Rev Allergy Immunol 2015;48:226-235.

16 Qiu D, Wang Q, Wang $\mathrm{H}$, et al. Validation of the simplified criteria for diagnosis of autoimmune hepatitis in Chinese patients. J Hepatol 2011;54: 340-347.

17 Lesna M, Hamlyn AN, Watson AJ. Intrahepatocytic copper and copper-binding protein in primary biliary cirrhosis: discrimination from chronic active hepatitis by means of orcein and rubeanic acid techniques. Digestion 1981;22:113-118.

18 Mounajjed T, Oxentenko AS, Qureshi H, et al. Revisiting the topic of histochemically detectable copper in various liver diseases with special focus on venous outflow impairment. Am J Clin Pathol 2013;139:79-86.

19 Van Eyken P, Sciot R, Desmet VJ. A cytokeratin immunohistochemical study of cholestatic liver disease: evidence that hepatocytes can express 'bile duct-type' cytokeratins. Histopathology 1989;15:125-135.
20 Rubio CA. Qualitative and quantitative differences between bile ducts in chronic hepatitis and in primary biliary cirrhosis. J Clin Pathol 2000;53:765-769.

21 Goldstein NS, Soman A, Gordon SC. Portal tract eosinophils and hepatocyte cytokeratin 7 immunoreactivity helps distinguish early-stage, mildly active primary biliary cirrhosis and autoimmune hepatitis. Am J Clin Pathol 2001;116:846-853.

22 Lindor KD, Gershwin ME, Poupon R, et al. American Association for Study of Liver Diseases: primary biliary cirrhosis. Hepatology 2009;50:291-308.

23 Ishak K, Baptista A, Bianchi L, et al. Histological grading and staging of chronic hepatitis. J Hepatol 1995 Jun;22:696-699.

24 Batts K, Ludwig J. Chronic hepatitis. An update on terminology and reporting. Am J Surg Pathol 1995;19: 1409-1417.

25 Manns MP, Czaja AJ, Gorham JD, et al. American Association for the Study of Liver Diseases. Diagnosis and management of autoimmune hepatitis. Hepatology 2010;51:2193-2213.

26 European Association for the Study of the Liver. EASL Clinical Practice Guidelines: autoimmune hepatitis. J Hepatol 2015;63:971-1004.

27 Kumari N, Kathuria R, Srivastav A, et al. Significance of histopathological features in differentiating autoimmune liver disease from nonautoimmune chronic liver disease in children. Eur J Gastroenterol Hepatol 2013;25:333-337.

28 Zen Y, Notsumata K, Tanaka N, Nakanuma Y. Hepatic centrilobular zonal necrosis with positive antinuclear antibody: a unique subtype or early disease of autoimmune hepatitis? Hum Pathol 2007;38:1669-1675.

29 Aizawa Y, Abe H, Sugita T, et al. Centrilobular zonal necrosis as a hallmark of a distinctive subtype of autoimmune hepatitis. Eur J Gastroenterol Hepatol 2016;28:391-397. 